\title{
Volumes of flow polytopes related to caracol graphs
}

\author{
Jihyeug Jang Jang Soo Kim* \\ Department of Mathematics \\ Sungkyunkwan University (SKKU) \\ Suwon, Gyeonggi-do 16419, South Korea \\ $\{$ ab4242, jangsookim\}@skku. edu
}

Submitted: Dec 16, 2019; Accepted: Sep 15, 2020; Published: Oct 30, 2020

(C) The authors. Released under the CC BY-ND license (International 4.0).

\begin{abstract}
Recently, Benedetti et al. introduced an Ehrhart-like polynomial associated to a graph. This polynomial is defined as the volume of a certain flow polytope related to a graph and has the property that the leading coefficient is the volume of the flow polytope of the original graph with net flow vector $(1,1, \ldots, 1)$. Benedetti et al. conjectured a formula for the Ehrhart-like polynomial of what they call a caracol graph. In this paper their conjecture is proved using constant term identities, labeled Dyck paths, and a cyclic lemma.
\end{abstract}

Mathematics Subject Classifications: 05A15, 05C21, 52B20

\section{Introduction}

The main objects in this paper are flow polytopes, which are certain polytopes associated to acyclic directed graphs with net flow vectors. Flow polytopes have interesting connections with representation theory, geometry, analysis, and combinatorics. A well known flow polytope is the Chan-Robbins-Yuen polytope, which is the flow polytope of the complete graph $K_{n+1}$ with net flow vector $(1,0, \ldots, 0)$. Chan, Robbins, and Yuen [4] conjectured that the volume of this polytope is a product of Catalan numbers. Their conjecture was proved by Zeilberger [15] using the Morris constant term identity [11], which is equivalent to the famous Selberg integral [12].

Since the discovery of the Chan-Robbins-Yuen polytope, researchers have found many flow polytopes whose volumes have nice product formulas, see $[3,5,6,8,9,10,14]$ and references therein. In this paper we add another flow polytope to this list by proving a product formula for the volume of the flow polytope coming from a caracol graph, which

*The second was supported by NRF grants \#2019R1F1A1059081 and \#2016R1A5A1008055. 


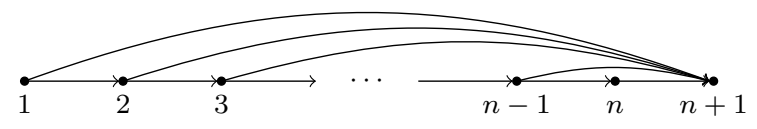

Figure 1: The Pitman-Stanley graph $\mathrm{PS}_{n+1}$.

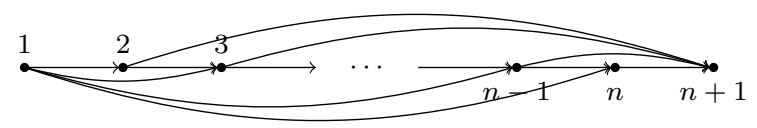

Figure 2: The caracol graph $\mathrm{Car}_{n+1}$.

was recently conjectured by Benedetti et al. [3]. In order to state our results we introduce necessary definitions.

We let $[n]:=\{1,2, \ldots, n\}$. Throughout this paper, we only consider connected directed graphs in which every vertex is an integer and every directed edge is of the form $(i, j)$ with $i<j$.

Let $G$ be a directed graph on vertex set $[n+1]$ with $m$ directed edges. We allow $G$ to have multiple edges but no loops. Let $\mathbf{a}=\left(a_{1}, a_{2}, \ldots, a_{n}\right) \in \mathbb{Z}^{n}$. An $m$-tuple $\left(b_{i j}\right)_{(i, j) \in E} \in \mathbb{R}_{\geqslant 0}^{m}$ is called an a-flow of $G$ if

$$
\sum_{(i, j) \in E} b_{i j}\left(\mathbf{e}_{i}-\mathbf{e}_{j}\right)=\left(a_{1}, \ldots, a_{n},-\sum_{i=1}^{n} a_{i}\right),
$$

where $\mathbf{e}_{i}$ is the standard basis vector in $\mathbb{R}^{n+1}$ with a one in the $i$ th entry and zeroes elsewhere. The flow polytope $\mathcal{F}_{G}(\mathbf{a})$ of $G$ with net flow $\mathbf{a}$ is defined as the set of all a-flows of $G$.

In this paper we consider the following two graphs, see Figures 1 and 2:

- The Pitman-Stanley graph $\mathrm{PS}_{n+1}$ is the graph with vertex set $[n+1]$ and edge set

$$
\{(i, i+1): i=1,2, \ldots, n\} \cup\{(i, n+1): i=1,2, \ldots, n-1\} .
$$

- The caracol graph $\mathrm{Car}_{n+1}$ is the graph with vertex set $[n+1]$ and edge set

$$
\{(i, i+1): i=1,2, \ldots, n\} \cup\{(1, i): i=3,4, \ldots, n\} \cup\{(i, n+1): i=2,3, \ldots, n-1\} .
$$

We note that the flow polytope $\mathcal{F}_{\mathrm{PS}_{n+1}}\left(a_{1}, \ldots, a_{n}\right)$ is affinely equivalent to the polytope $\Pi_{n-1}\left(a_{1}, \ldots, a_{n-1}\right):=\left\{\left(x_{1}, \ldots, x_{n-1}\right): x_{i} \geqslant 0, x_{1}+\cdots+x_{i} \leqslant a_{1}+\cdots+a_{i}, 1 \leqslant i \leqslant n-1\right\}$, considered in [13]. Pitman and Stanley [13] found volume formulas for certain polytopes, 
which can be restated as normalized volumes of flow polytopes as follows:

$$
\begin{aligned}
\operatorname{vol} \mathcal{F}_{\mathrm{PS}_{n+1}}\left(a, b^{n-2}, d\right) & =a(a+(n-1) b)^{n-2}, \\
\operatorname{vol} \mathcal{F}_{\mathrm{PS}_{n+1}}\left(a, b^{n-3}, c, d\right) & =a(a+(n-1) b)^{n-2}+(n-1) a(c-b)(a+(n-2) b)^{n-3}, \\
\operatorname{vol} \mathcal{F}_{\mathrm{PS}_{n+1}}\left(a, b^{n-m-2}, c, 0^{m-1}, d\right) & =a \sum_{j=0}^{m}\left(\begin{array}{c}
n \\
j
\end{array}\right)(c-(m+1-j) b)^{j}(a+(n-1-j) b)^{n-j-2},
\end{aligned}
$$

where $b^{k}$ means the sequence $b, b, \ldots, b$ of $k b$ 's. We note that vol $\mathcal{F}_{\mathrm{PS}_{n+1}}\left(a_{1}, \ldots, a_{n}\right)$ is independent of $a_{n}$.

In [3], Benedetti et al. introduced combinatorial models called gravity diagrams and unified diagrams to compute volumes of flow polytopes. Using these models they showed

$$
\begin{aligned}
\operatorname{vol} \mathcal{F}_{\operatorname{Car}_{n+1}}\left(a^{n}\right) & =C_{n-2} a^{n} n^{n-2}, \\
\operatorname{vol} \mathcal{F}_{\operatorname{Car}_{n+1}}\left(a, b^{n-1}\right) & =C_{n-2} a^{n-2}(a+(n-1) b)^{n-2},
\end{aligned}
$$

where $C_{k}:=\frac{1}{k+1}\left(\begin{array}{c}2 k \\ k\end{array}\right)$ is the $k$ th Catalan number.

For a positive integer $k$ and a directed graph $G$ on $[n+1]$, let $\widehat{G}(k)$ be the directed graph obtained from $G$ by adding a new vertex 0 and $k$ multiple edges $(0, i)$ for each $1 \leqslant i \leqslant n$. Then we define

$$
E_{G}(k)=\operatorname{vol} \mathcal{F}_{\widehat{G}(k)}\left(1,0^{n}\right) .
$$

In [3], Benedetti et al. showed that $E_{G}(k)$ is a polynomial function in $k$. Therefore we can consider the polynomial $E_{G}(x)$. They also showed that these polynomials $E_{G}(x)$ have similar properties as Ehrhart polynomials. For example, the leading coefficient of $E_{G}(x)$ is the normalized volume of $\mathcal{F}_{G}\left(1^{n}\right)$. For this reason, they called $E_{G}(x)$ an Ehrhart-like polynomial. In the same paper they proved the following theorem.

Theorem 1. We have

$$
E_{\mathrm{PS}_{n+1}}(k)=\frac{1}{k n-1}\left(\begin{array}{c}
(k+1) n-2 \\
n
\end{array}\right)
$$

Our main result is the following theorem, which was conjectured in [3].

Theorem 2. We have

$$
E_{\mathrm{Car}_{n+1}}(k)=\frac{1}{k n+n-3}\left(\begin{array}{c}
k n+2 n-5 \\
n-1
\end{array}\right)\left(\begin{array}{c}
n+k-3 \\
k-1
\end{array}\right) .
$$

In this paper we prove Theorem 2 and give an alternative proof of Theorem 1.

The remainder of this paper is organized as follows. In Section 2 we use the Lidskii volume formula to interpret $E_{\mathrm{Car}_{n+1}}(k)$ as a Kostant partition function, which is equal to the constant term of a Laurent series. In Section 3 we introduce labeled Dyck paths 
and show that the constant term is equal to the number of certain labeled Dyck paths. In Section 4 we enumerate these labeled Dyck paths using a cyclic lemma. In Section 5 using our combinatorial models we show the following volume formulas:

$$
\begin{gathered}
\operatorname{vol} \mathcal{F}_{\mathrm{PS}_{n+1}}\left(a, b, c^{n-2}\right)=(a+b-c)(a+b+(n-2) c)^{n-2}+(-b+c)(b+(n-2) c)^{n-2} \\
\begin{aligned}
\operatorname{vol} \mathcal{F}_{\mathrm{PS}_{n+1}}\left(a, b, c, d^{n-3}\right)=(a+b & +c-2 d)(a+b+c+(n-3) d)^{n-2} \\
& -(b+c-2 d)(b+c+(n-3) d)^{n-2} \\
& \quad-(n-1) a(c-d)(c+(n-3) d)^{n-3} \\
\operatorname{vol} \mathcal{F}_{\mathrm{Car}_{n+1}}\left(a, b, c^{n-2}\right)= & C_{n-2} a^{n-1}(a+b(n-1))(a+b+c(n-2))^{n-3}
\end{aligned}
\end{gathered}
$$

where (9) was conjectured by Benedetti et al. in [3].

\section{Constant term identities}

In this section we review the Lidskii volume formula and restate Theorems 1 and 2 as constant term identities.

Let $G$ be a directed graph on $[n+1]$ and define $\mathbf{a}^{\prime}=\left(a_{1}, \ldots, a_{n},-\sum_{i=1}^{n} a_{i}\right)$ for $\mathbf{a}=$ $\left(a_{1}, \ldots, a_{n}\right) \in \mathbb{Z}^{n}$. The Kostant partition function $K_{G}\left(\mathbf{a}^{\prime}\right)$ of $G$ at $\mathbf{a}^{\prime}$ is the number of integer points of $\mathcal{F}_{G}(\mathbf{a})$, i.e., if $G$ has $m$ edges,

$$
K_{G}\left(\mathbf{a}^{\prime}\right)=\left|\mathcal{F}_{G}(\mathbf{a}) \cap \mathbb{Z}^{m}\right|
$$

We denote by $\left.G\right|_{n}$ the restriction of $G$ to the vertices in $[n]$. Let outdeg $(i)$ denote the out-degree of vertex $i$ in $G$. The following formula, known as the Lidskii volume formula, allows us to express the (normalized) volume of the flow polytope $\mathcal{F}_{G}(\mathbf{a})$ in terms of Kostant partition functions, see [2, Theorem 38].

Theorem 3 (Lidskii volume formula). Let $G$ be a connected directed graph on $[n+1]$ with $m$ directed edges, where every directed edge is of the form $(i, j)$ with $i<j$ and let $\mathbf{a}=$ $\left(a_{1}, a_{2}, \ldots, a_{n}\right) \in \mathbb{Z}^{n}$. Denoting $\mathbf{t}=\left(t_{1}, \ldots, t_{n}\right):=\left(\operatorname{outdeg}_{G}(1)-1, \ldots, \operatorname{outdeg}_{G}(n)-1\right)$, we have

$$
\operatorname{vol} \mathcal{F}_{G}(\mathbf{a})=\sum_{\substack{|\mathbf{s}|=m-n \\
\mathbf{s} \geqslant \mathbf{t}}}\left(\begin{array}{c}
m-n \\
s_{1}, s_{2}, \ldots, s_{n}
\end{array}\right) a_{1}^{s_{1}} \ldots a_{n}^{s_{n}} K_{\left.G\right|_{n}}(\mathbf{s}-\mathbf{t}),
$$

where the sum is over all sequences $\mathbf{s}=\left(s_{1}, \ldots, s_{n}\right)$ of nonnegative integers such that $|\mathbf{s}|=s_{1}+\cdots+s_{n}=m-n$ and $\mathbf{s} \geqslant \mathbf{t}$ in dominance order, i.e., $\sum_{i=1}^{k} s_{i} \geqslant \sum_{i=1}^{k} t_{i}$ for $k=1,2, \ldots, n$.

In this paper, we use both expressions $K_{\left.G\right|_{n}}\left(a_{1}, \ldots, a_{n}\right)$ and $K_{G}\left(a_{1}, \ldots, a_{n}, 0\right)$ together. Note that if $\mathbf{a}=\left(1,0^{n-1}\right)$ in Theorem 3 there is only one nonzero term in the sum giving the following corollary, see [2], [13], or [7, Corollary 1.4]. 
Corollary 4. For a directed graph $G$ on $[n+1]$, we have

$$
\operatorname{vol} \mathcal{F}_{G}\left(1,0^{n-1}\right)=K_{G}\left(p, 1-\operatorname{outdeg}_{G}(2), 1-\operatorname{outdeg}_{G}(3), \ldots, 1-\operatorname{outdeg}_{G}(n), 0\right),
$$

where $p=\operatorname{outdeg}_{G}(2)+\operatorname{outdeg}_{G}(3)+\cdots+\operatorname{outdeg}_{G}(n)-n+1$.

For a multivariate rational function $f\left(x_{1}, x_{2}, \ldots, x_{n}\right)$ we denote by $\mathrm{CT}_{x_{i}} f$ the constant term of the Laurant series expansion of $f$ with respect to $x_{i}$ by considering other variables as constants. Since $\mathrm{CT}_{x_{1}} f$ is a rational function in $x_{2}, \ldots, x_{n}$, we can apply $\mathrm{CT}_{x_{2}}$ to it. Repeating in this way the constant term $\mathrm{CT}_{x_{n}} \ldots \mathrm{CT}_{x_{1}} f$ is defined. We also define $\left[x_{n}^{a_{n}} \ldots x_{1}^{a_{1}}\right] f$ to be the coefficient of the monomial $x_{n}^{a_{n}} \ldots x_{1}^{a_{1}}$ in the Laurent expansion of $f$ when expanded in the variables $x_{1}, x_{2}, \ldots, x_{n}$ in this order. Note that we have

$$
\left[x_{n}^{a_{n}} \ldots x_{1}^{a_{1}}\right] f=\mathrm{CT}_{x_{n}} \ldots \mathrm{CT}_{x_{1}}\left(x_{n}^{-a_{n}} \ldots x_{1}^{-a_{1}} f\right) \text {. }
$$

Let $G$ be a directed graph on $[n+1]$. Then for $\mathbf{a}=\left(a_{1}, \ldots, a_{n}\right) \in \mathbb{Z}^{n}$ and $a_{n+1}=$ $-\left(a_{1}+\cdots+a_{n}\right)$, the Kostant partition function $K_{G}\left(\mathbf{a}^{\prime}\right)$ can be computed by

$$
K_{G}\left(\mathbf{a}^{\prime}\right)=\left[x_{n+1}^{a_{n+1}} \cdots x_{1}^{a_{1}}\right] \prod_{(i, j) \in E(G)}\left(1-\frac{x_{i}}{x_{j}}\right)^{-1} .
$$

Now we are ready to express $E_{\mathrm{PS}_{n+1}}(k)$ and $E_{\mathrm{Car}_{n+1}}(k)$ as constant terms of Laurent series. Throughout this paper the factor $\left(x_{j}-x_{i}\right)^{-1}$, where $i<j$, means the Laurent expansion

$$
\left(x_{j}-x_{i}\right)^{-1}=\frac{1}{x_{j}}\left(1-\frac{x_{i}}{x_{j}}\right)^{-1}=\frac{1}{x_{j}} \sum_{l \geqslant 0}\left(\frac{x_{i}}{x_{j}}\right)^{l} .
$$

Proposition 5. We have

$$
\begin{aligned}
& E_{\mathrm{PS}_{n+1}}(k)=\mathrm{CT}_{x_{n}} \ldots \mathrm{CT}_{x_{1}} \prod_{i=1}^{n}\left(1-x_{i}\right)^{-k} \prod_{i=1}^{n-1}\left(x_{i+1}-x_{i}\right)^{-1}, \\
& E_{\mathrm{Car}_{n+2}}(k)=\mathrm{CT}_{x_{n}} \ldots \mathrm{CT}_{x_{1}} \frac{1}{x_{1}} \prod_{i=1}^{n}\left(1-x_{i}\right)^{-k} \prod_{i=1}^{n-1}\left(x_{n}-x_{i}\right)^{-1} \prod_{i=1}^{n-2}\left(x_{i+1}-x_{i}\right)^{-1} .
\end{aligned}
$$

Proof. We will only prove (14) since (13) can be proved similarly. Let $G=\mathrm{Car}_{n+1}$ and $H=\widehat{G}(k)$. Then $H$ is a graph with vertices $0,1,2, \ldots, n+1$, and by (6) and (10),

$$
E_{\operatorname{Car}_{n+1}}(k)=K_{H}\left(p, 1-\operatorname{outdeg}_{H}(1), 1-\operatorname{outdeg}_{H}(2), \ldots, 1-\operatorname{outdeg}_{H}(n), 0\right),
$$

where $p=\operatorname{outdeg}_{H}(1)+\operatorname{outdeg}_{H}(2)+\cdots+\operatorname{outdeg}_{H}(n)-n$. Since outdeg $(1)=n-1$, $\operatorname{outdeg}_{H}(n)=1$, and $\operatorname{outdeg}_{H}(i)=2$ for $2 \leqslant i \leqslant n-1$, we can rewrite the above equation as

$$
E_{\mathrm{Car}_{n+1}}(k)=K_{H}\left(2 n-4,2-n,(-1)^{n-2}, 0,0\right) .
$$


Then, by (12), we obtain

$$
E_{\mathrm{Car}_{n+1}}(k)=\left[x_{n-1}^{-1} \cdots x_{2}^{-1} x_{1}^{2-n} x_{0}^{2 n-4}\right] \prod_{(i, j) \in E(H)}\left(1-\frac{x_{i}}{x_{j}}\right)^{-1} .
$$

Since every term in the expansion of

$$
\prod_{(i, j) \in E(H)}\left(1-\frac{x_{i}}{x_{j}}\right)^{-1}=\prod_{i=1}^{n}\left(1-\frac{x_{0}}{x_{i}}\right)^{-k}\left(\prod_{i=2}^{n}\left(1-\frac{x_{1}}{x_{i}}\right)\left(1-\frac{x_{i}}{x_{n+1}}\right) \prod_{i=2}^{n-1}\left(1-\frac{x_{i}}{x_{i+1}}\right)\right)^{-1}
$$

is homogeneous of degree 0 in the variables $x_{0}, x_{1}, \ldots, x_{n+1}$, we can set $x_{0}=1$ in $(15)$. Moreover, since every term in the expansion of $\left(1-x_{i} / x_{n+1}\right)^{-1}$ has a negative power of $x_{n+1}$ except for the constant term 1, we can omit the factors involving $x_{n+1}$ in (15). Then, by the same argument, we can also omit the factors involving $x_{n}$ in (15) to obtain

$$
E_{\mathrm{Car}_{n+1}}(k)=\left[x_{1}^{2-n} x_{2}^{-1} \cdots x_{n-1}^{-1}\right] \prod_{i=1}^{n-1}\left(1-\frac{1}{x_{i}}\right)^{-k} \prod_{i=2}^{n-1}\left(1-\frac{x_{1}}{x_{i}}\right)^{-1} \prod_{i=2}^{n-2}\left(1-\frac{x_{i}}{x_{i+1}}\right)^{-1} .
$$

By replacing $x_{j}$ by $x_{n-j}^{-1}$ for each $1 \leqslant j \leqslant n-1$ we have

$$
E_{\mathrm{Car}_{n+1}}(k)=\left[x_{n-1}^{n-2} x_{n-2} \cdots x_{1}\right] \prod_{i=1}^{n-1}\left(1-x_{i}\right)^{-k} \prod_{i=1}^{n-2}\left(1-\frac{x_{i}}{x_{n-1}}\right)^{-1} \prod_{i=1}^{n-3}\left(1-\frac{x_{i}}{x_{i+1}}\right)^{-1},
$$

which is equivalent to (14) by (11).

By Proposition 5, we can restate Theorems 1 and 2 as follows.

Theorem 6. We have

$$
\mathrm{CT}_{x_{n}} \ldots \mathrm{CT}_{x_{1}} \prod_{i=1}^{n}\left(1-x_{i}\right)^{-k} \prod_{i=1}^{n-1}\left(x_{i+1}-x_{i}\right)^{-1}=\frac{1}{k n-1}\left(\begin{array}{c}
(k+1) n-2 \\
n
\end{array}\right) .
$$

Theorem 7. We have

$$
\begin{aligned}
\mathrm{CT}_{x_{n}} \ldots \mathrm{CT}_{x_{1}} \frac{1}{x_{1}} \prod_{i=1}^{n}\left(1-x_{i}\right)^{-k} & \prod_{i=1}^{n-1}\left(x_{n}-x_{i}\right)^{-1} \prod_{i=1}^{n-2}\left(x_{i+1}-x_{i}\right)^{-1} \\
& =\frac{1}{k(n+1)+n-2}\left(\begin{array}{c}
k n+k+2 n-3 \\
n
\end{array}\right)\left(\begin{array}{c}
n+k-2 \\
k-1
\end{array}\right) .
\end{aligned}
$$




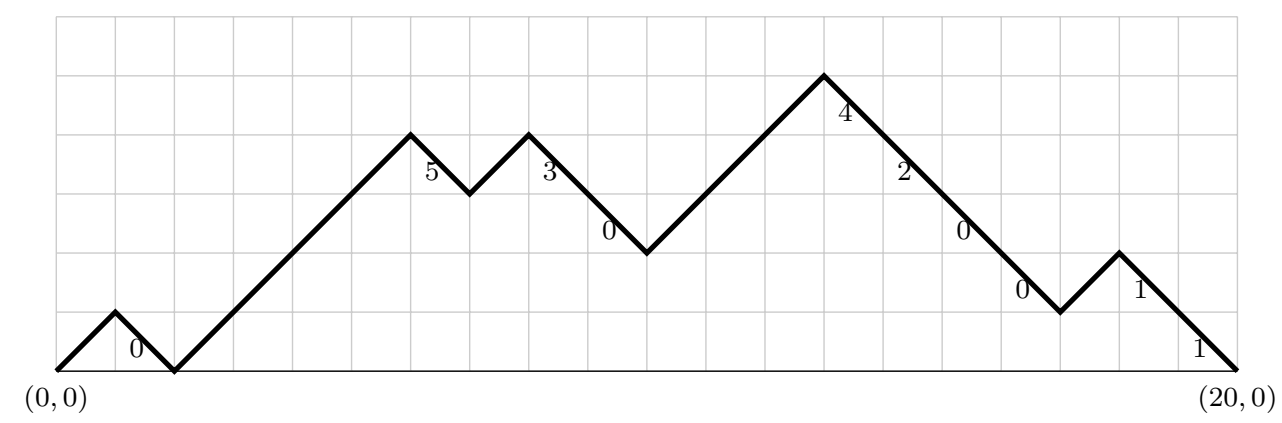

Figure 3: A 5-labeled Dyck path of length 20. The labels of every consecutive down-steps must be in weakly decreasing order.

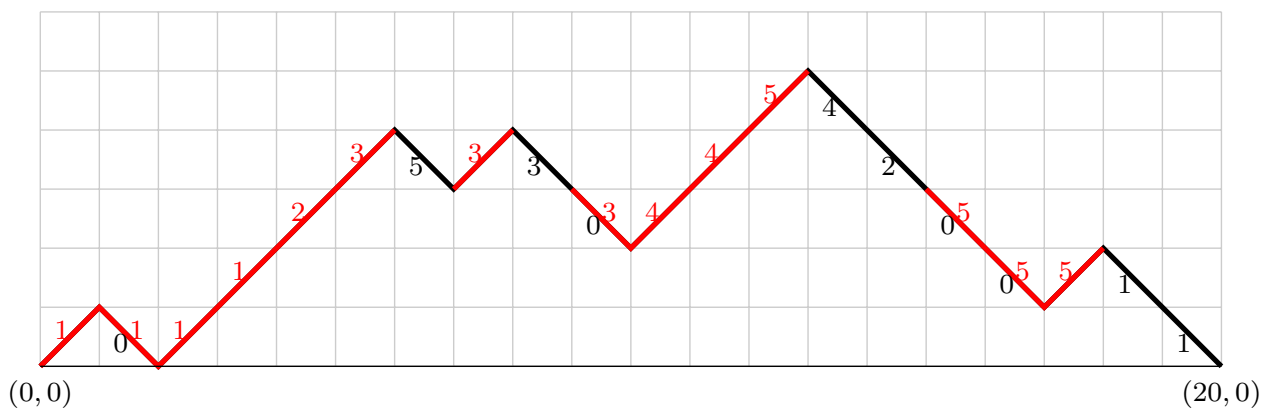

Figure 4: A doubly 5-labeled Dyck path of length 20. The down-steps labeled 0 and the up-steps are the red steps and their additional labels are written in red. The red labels must be in weakly increasing order.

\section{Labeled Dyck Paths}

In this section we give combinatorial meanings to the constant terms in Theorems 6 and 7 using labeled Dyck paths.

A Dyck path of length $2 n$ is a lattice path from $(0,0)$ to $(2 n, 0)$ consisting of up-steps $(1,1)$ and down-steps $(1,-1)$ lying on or above the line $y=0$. The set of Dyck paths of length $2 n$ is denoted by Dyck $_{n}$.

Let $k$ be a positive integer. A $k$-labeled Dyck path is a Dyck path with a labeling on the down-steps such that the label of each down-step is an integer $0 \leqslant i \leqslant k$ and the labels of any consecutive down-steps are in weakly decreasing order, see Figure 3. A doubly $k$-labeled Dyck path is a $k$-labeled Dyck path together with an additional labeling on the down-steps labeled 0 and the up-steps with integers from $\{1,2, \ldots, k\}$ such that the additional labels on these steps are weakly increasing, see Figure 4.

We denote by $\operatorname{Dyck}_{n}(k)$ (resp. Dyck ${ }_{n}^{(2)}(k)$ ) the set of $k$-labeled Dyck paths (resp. doubly $k$-labeled Dyck paths) of length $2 n$. We also denote by $\operatorname{Dyck}_{n}(k, d)$ the set of $k$-labeled Dyck paths of length $2 n$ with exactly $d$ down-steps labeled 0 .

A multiset is a set with repetitions allowed. Let $\left(\left(\begin{array}{c}n \\ m\end{array}\right)\right):=\left(\begin{array}{c}n+m-1 \\ m\end{array}\right)$. Then $\left(\left(\begin{array}{c}n \\ m\end{array}\right)\right)$ is the number of multisets with $m$ elements taken from $[n]$. Equivalently, $\left(\left(\begin{array}{c}n \\ m\end{array}\right)\right)$ is the number of 
nonnegative integer solutions $\left(a_{1}, a_{2}, \ldots, a_{n}\right)$ to $a_{1}+a_{2}+\cdots+a_{n}=m$ and also the number of $m$-tuples $\left(i_{1}, i_{2}, \ldots, i_{m}\right)$ of nonnegative integers satisfying $1 \leqslant i_{1} \leqslant i_{2} \leqslant \ldots \leqslant i_{m} \leqslant n$. The following proposition is immediate from the definitions of $\operatorname{Dyck}_{n}^{(2)}(k)$ and $\operatorname{Dyck}_{n}(k, d)$.

Proposition 8. We have

$$
\left|\operatorname{Dyck}_{n}^{(2)}(k)\right|=\sum_{d=0}^{n}\left|\operatorname{Dyck}_{n}(k, d)\right|\left(\left(\begin{array}{c}
k \\
n+d
\end{array}\right)\right) .
$$

We now show that the constant terms in Theorems 6 and 7 have the following combinatorial interpretations.

Theorem 9. We have

$$
\mathrm{CT}_{x_{n}} \ldots \mathrm{CT}_{x_{1}} \prod_{i=1}^{n}\left(1-x_{i}\right)^{-k} \prod_{i=1}^{n-1}\left(x_{i+1}-x_{i}\right)^{-1}=\left|\operatorname{Dyck}_{n-1}(k, 0)\right| \text {. }
$$

Proof. Consider that we choose $x_{i}^{a_{i 1}} x_{i}^{a_{i 2}} \cdots x_{i}^{a_{i k}}$ in $\left(1-x_{i}\right)^{-k}=\left(1+x_{i}+x_{i}^{2}+\cdots\right) \cdots(1+$ $\left.x_{i}+x_{i}^{2}+\cdots\right)$ for $i=1,2, \ldots, n$ and we choose $x_{i}^{b_{i}} / x_{i+1}^{b_{i}+1}$ in $\left(x_{i+1}-x_{i}\right)^{-1}=1 / x_{i+1}+$ $x_{i} / x_{i+1}^{2}+x_{i}^{2} / x_{i+1}^{3}+\cdots$ for $i=1,2, \ldots, n-1$. Then $\prod_{i=1}^{n}\left(1-x_{i}\right)^{-k} \prod_{i=1}^{n-1}\left(x_{i+1}-x_{i}\right)^{-1}=$ $\sum \prod_{i=1}^{n}\left(x_{i}^{a_{i 1}+\cdots+a_{i k}+b_{i}-b_{i-1}-1}\right)$, where the sum is over all nonnegative integers $a_{i j}, b_{i}$ for $1 \leqslant i \leqslant n$ and $1 \leqslant j \leqslant k$ with $b_{0}=-1$ and $b_{n}=0$. Hence the left-hand side is the number of the nonnegative integer solutions to the equations $a_{i 1}+\cdots+a_{i k}=b_{i-1}-b_{i}+1$ for $i=1,2, \ldots, n$ with $b_{0}=-1, b_{n}=0$. If we set $r_{i}=b_{i-1}-b_{i}+1$, so that $r_{1}+r_{2}+\cdots+r_{j}=$ $j+b_{0}-b_{j} \leqslant j-1$, then the number of solutions is $\sum \prod_{i=1}^{n}\left(\left(\begin{array}{c}k \\ r_{i}\end{array}\right)\right)$, where the sum is over all nonnegative integers $r_{1}, \ldots, r_{n}$ with $r_{1}+\cdots+r_{j} \leqslant j-1$ for $j=1,2, \ldots, n-1$ and $r_{1}+\cdots+r_{n}=n-1$. For such an $n$-tuple $\left(r_{1}, \ldots, r_{n}\right)$, let $D$ be the Dyck path of length $2(n-1)$ such that the number of consecutive down-steps after the $i$ th up-step is $r_{i+1}$ for $i=1, \ldots, n-1$. The $\operatorname{map}\left(r_{1}, \ldots, r_{n}\right) \mapsto D$ is a bijection from the set of $n$-tuples satisfying the above conditions to Dyck $_{n-1}$. Under this correspondence, $\prod_{i=1}^{n}\left(\left(\begin{array}{c}k \\ r_{i}\end{array}\right)\right)$ is the number of $k$-labeled Dyck paths in $\operatorname{Dyck}_{n-1}(k, 0)$ whose underlying Dyck path is $D$. Therefore we obtain the result.

Theorem 10. We have

$$
\mathrm{CT}_{x_{n}} \ldots \mathrm{CT}_{x_{1}} \frac{1}{x_{1}} \prod_{i=1}^{n}\left(1-x_{i}\right)^{-k} \prod_{i=1}^{n-1}\left(x_{n}-x_{i}\right)^{-1} \prod_{i=1}^{n-2}\left(x_{i+1}-x_{i}\right)^{-1}=\left|\operatorname{Dyck}_{n-1}^{(2)}(k)\right| \text {. }
$$

Proof. Similarly to the previous theorem, considering $x_{i}^{a_{i 1}} x_{i}^{a_{i 2}} \cdots x_{i}^{a_{i k}}$ in $\left(1-x_{i}\right)^{-k}$ and $x_{i}^{b_{i}} / x_{i+1}^{b_{i}+1}$ in $\left(x_{i+1}-x_{i}\right)^{-1}$ and $x_{i}^{c_{i}} / x_{n}^{c_{i}+1}$ in $\left(x_{n}-x_{i}\right)^{-1}$, we get that the left-hand side is the number of the nonnegative integer solutions to the equations $a_{i 1}+\cdots+a_{i k}+b_{i}+c_{i}=1+b_{i-1}$ for $i=1,2, \ldots, n-1$ and $a_{n 1}+\cdots+a_{n k}+b_{n}+c_{n}=n-1+c_{1}+\cdots+c_{n-1}$ with $b_{0}=b_{n-1}=b_{n}=c_{n}=0$. If we set $r_{i}=b_{i-1}-b_{i}+1$ for $i=1,2, \ldots, n-1$, then the number of solutions is $\sum_{r_{i}, c_{i}} \prod_{i=1}^{n-1}\left(\left(\begin{array}{c}k \\ r_{i}-c_{i}\end{array}\right)\right)\left(\left(\begin{array}{c}k \\ n-1+c_{1}+\cdots+c_{n-1}\end{array}\right)\right)$ where the sum is over all nonnegative integers $r_{i}, c_{i}$ for $i=1,2, \ldots, n-1$ with $r_{1}+\cdots+r_{j} \leqslant j$ for $j=1,2, \ldots, n-2$ 
and $r_{1}+\cdots+r_{n-1}=n-1$. For such an $n$-tuple $\left(r_{1}, \ldots, r_{n}\right)$, let $D$ be the Dyck path of length $2(n-1)$ such that the number of consecutive down-steps after the $i$ th up-step is $r_{i}$ for $i=1, \ldots, n-1$. The map $\left(r_{1}, \ldots, r_{n}\right) \mapsto D$ is a bijection from the set of $n$-tuples satisfying the above conditions to Dyck ${ }_{n-1}$. Regard $\left(\left(\begin{array}{c}k \\ r-c\end{array}\right)\right)$ as the number of $r$-tuples $\left(i_{1}, \ldots, i_{r}\right)$ of integers with $k \geqslant i_{1} \geqslant \ldots \geqslant i_{r-c} \geqslant 1$ and $i_{r-c+1}=\cdots=i_{r}=0$. Then $\sum_{c_{i}} \prod_{i=1}^{n-1}\left(\left(\begin{array}{c}k \\ r_{i}-c_{i}\end{array}\right)\right)\left(\left(\begin{array}{c}k \\ n-1+c_{1}+\cdots+c_{n-1}\end{array}\right)\right)$, where the sum is over all nonnegative integers $c_{i}$ for $i=1,2, \ldots, n-1$ with $c_{i} \leqslant r_{i}$, is the number of doubly $k$-labeled Dyck paths whose underlying Dyck path is $D$. Therefore we obtain the result.

Note that by Proposition 8, we can compute the constant terms in Theorems 9 and 10 if we have a formula for the cardinality $\left|\operatorname{Dyck}_{n}(k, d)\right|$. Therefore our next step is to find this number.

\section{A cyclic lemma}

Let $\operatorname{Dyck}_{n}\left(k ; a_{0}, a_{1}, \ldots, a_{k}\right)$ denote the set of $k$-labeled Dyck paths of length $2 n$ such that the number of down-steps with label $i$ is $a_{i}$ for $0 \leqslant i \leqslant k$. In this section we prove the following theorem using a cyclic lemma.

Theorem 11. We have

$$
\left|\operatorname{Dyck}_{n}\left(k ; a_{0}, a_{1}, \ldots, a_{k}\right)\right|=\frac{1}{n+1} \prod_{i=0}^{k}\left(\left(\begin{array}{c}
n+1 \\
a_{i}
\end{array}\right)\right) .
$$

Remark 12. A parking function of length $n$ is a tuple $\left(p_{1}, p_{2}, \ldots, p_{n}\right) \in \mathbb{Z}_{>0}^{n}$ with a condition that $q_{i} \leqslant i$ for $i=1,2, \ldots, n$ where $\left(q_{1}, q_{2}, \ldots, q_{n}\right)$ is the rearrangement of $\left(p_{1}, p_{2}, \ldots, p_{n}\right)$ in weakly increasing order. Let $P F_{n}$ be the set of parking function of length $n$. There is a well-known bijection between $P F_{n}$ and $n$-labeled Dyck paths of length $2 n$ which the number of each label from 1 to $n$ equals 1 . Thus, using Theorem 11 , we have

$$
\left|P F_{n}\right|=\left|\operatorname{Dyck}_{n}(n ; 0,1,1, \ldots, 1)\right|=(n+1)^{n-1} .
$$

Remark 13. Recently, Yip [14, Theorem 3.18] considered a set $\mathcal{T}_{k}(n, i)$ of certain labeled Dyck paths and found a simple formula for its cardinality using a cyclic lemma. Using our notation, this set can be written

$$
\mathcal{T}_{k}(n, i)=\bigcup_{a_{0}+\cdots+a_{k-1}=n-i} \operatorname{Dyck}_{n}\left(k+i-1 ; a_{0}, \ldots, a_{k-1}, 1^{i}\right)
$$

The proof of Theorem 11 in this section is essentially the same as that in $[14$, Theorem 3.18].

A $k$-labeled Dyck word of length $2 n$ is defined as a sequence $w=w_{1} \ldots w_{2 n}$ of letters in $\left\{U, D_{0}, D_{1}, \ldots, D_{k}\right\}$ satisfying the following conditions:

- The number of U's is equal to $n$. 
- For any prefix $w_{1} \ldots w_{j}$, the number of $U$ 's is greater than or equal to the total number of $D_{i}$ 's for $0 \leqslant i \leqslant k$.

- The labels of any consecutive $D_{i}$ 's are in weakly decreasing order, i.e., if $w_{i}=D_{a}$ and $w_{i+1}=D_{b}$, then $a \geqslant b$.

Replacing each up step by $U$ and each down step labeled $i$ by $D_{i}$ is an obvious bijection from $k$-labeled Dyck paths to $k$-labeled Dyck words. For example, the $k$-labeled Dyck word corresponding to the $k$-labeled Dyck path in Figure 3 is

$$
U D_{0} U U U U D_{5} U D_{3} D_{0} U U U D_{4} D_{2} D_{0} D_{0} U D_{1} D_{1}
$$

From now on, we will identify $k$-labeled Dyck paths with $k$-labeled Dyck words using this bijection. Note that $\operatorname{Dyck}_{n}\left(k ; a_{0}, a_{1}, \ldots, a_{k}\right)$ is then the set of $k$-labeled Dyck words of length $2 n$ in which the number of $D_{i}$ 's is equal to $a_{i}$ for $0 \leqslant i \leqslant k$. We can count such words by using a well-known cyclic argument. We first need another definition.

An extended $k$-labeled word of length $2 n+1$ is a sequence $w=w_{1} \ldots w_{2 n+1}$ of letters in $\left\{U, D_{0}, D_{1}, \ldots, D_{k}\right\}$ satisfying the following conditions:

- $w_{1}=U$.

- The number of $U$ 's is equal to $n+1$.

- The labels of any consecutive $D_{i}$ 's are in weakly decreasing order, i.e., if $w_{i}=D_{a}$ and $w_{i+1}=D_{b}$, then $a \geqslant b$.

Define $\mathrm{EW}_{n}(k)$ to be the set of all extended $k$-labeled words of length $2 n+1$. For $w=w_{1} \ldots w_{2 n+1} \in \mathrm{EW}_{n}(k)$, we define the integer index $(w)$ using the following algorithm. Here, $w=w_{1} \ldots w_{2 n+1}$ is cyclically ordered, which means that $w_{1}$ is followed by $w_{2}, w_{2}$ is followed by $w_{3}$, and so on, and $w_{2 n+1}$ is followed by $w_{1}$.

- Find a letter $U$ followed by a $D_{i}$ for some $0 \leqslant i \leqslant k$ in cyclic order and delete this pair $U$ and $D_{i}$ from $w$. Repeat this until there is only one letter left, which must be $U$.

- If the remaining $U$ is the $j \operatorname{th} U$ in the original word $w$ then $\operatorname{define} \operatorname{index}(w)=j$.

We also define the shifting operator $s: \mathrm{EW}_{n}(k) \rightarrow \mathrm{EW}_{n}(k)$ by

$$
s(w):=w_{i} w_{i+1} \cdots w_{2 n+1} w_{1} \ldots w_{i-1},
$$

where $i$ is the largest integer with $w_{i}=U$.

Example 14. Let $w=U D_{1} D_{0} U U D_{1} U \in \mathrm{EW}_{3}(1)$. Then by the algorithm,

$$
\underline{U D_{1}} D_{0} U U D_{1} U \rightarrow D_{0} U \underline{U D_{1}} U \rightarrow \underline{D_{0}} U \underline{U} \rightarrow U
$$

we get index $(w)=2$ since the remaining $U$ is the second $U$ in $w$. 
Since the above algorithm treats the word $w$ cyclically one can easily see that the following lemma holds.

Lemma 15. For any element $w \in \mathrm{EW}_{n}(k)$, we have

$$
\operatorname{index}(s(w)) \equiv \operatorname{index}(w)+1 \quad \bmod n+1 .
$$

Observe that for $w=w_{1} w_{2} \ldots w_{2 n+1} \in \operatorname{EW}_{n}(k)$ we have $w_{1} \ldots w_{2 n} \in \operatorname{Dyck}_{n}(k)$ if and only if $\operatorname{index}(w)=n+1$. Therefore, by Lemma 15, for each $w \in \mathrm{EW}_{n}(k)$ there is a unique integer $0 \leqslant j \leqslant n$ such that $s^{j}(w)=w^{\prime} U$ for some $k$-labeled Dyck word $w^{\prime}$ of length $2 n$. This defines a map $p: \mathrm{EW}_{n}(k) \rightarrow \operatorname{Dyck}_{n}(k)$ sending $w$ to $p(w)=w^{\prime}$. Again, by Lemma 15 , this is a $(n+1)$-to- 1 map. Note that $w$ and $w^{\prime}$ have the same the number of steps $D_{i}$ for each $0 \leqslant i \leqslant k$. We have proved the following proposition.

Proposition 16. There is an $(n+1)$-to-1 map $p: \mathrm{EW}_{n}(k) \rightarrow \operatorname{Dyck}_{n}(k)$ preserving the number of $D_{i}$ 's for $0 \leqslant i \leqslant k$.

We now can prove Theorem 11 easily.

Proof of Theorem 11. By Proposition 16, $(n+1)\left|\operatorname{Dyck}_{n}\left(k ; a_{0}, a_{1}, \ldots, a_{k}\right)\right|$ is the number of elements $w \in \mathrm{EW}_{n}(k)$ in which $D_{i}$ appears $a_{i}$ times for $0 \leqslant i \leqslant k$. Since consecutive $D_{i}$ 's are always ordered according to their subscripts, such elements $w$ are obtained from the sequence $U \ldots U$ of $n+1$ 's by inserting $a_{i} D_{i}$ 's after $U$ 's in $\left(\left(\begin{array}{c}n+1 \\ a_{i}\end{array}\right)\right)$ ways for $0 \leqslant i \leqslant k$ independently. Thus we have

$$
(n+1)\left|\operatorname{Dyck}_{n}\left(k ; a_{0}, a_{1}, \ldots, a_{k}\right)\right|=\prod_{i=0}^{k}\left(\left(\begin{array}{c}
n+1 \\
a_{i}
\end{array}\right)\right),
$$

which completes the proof.

As corollaries we obtain formulas for $\left|\operatorname{Dyck}_{n}(k, d)\right|$ and $\left|\operatorname{Dyck}_{n-1}^{(2)}(k)\right|$.

Corollary 17. We have

$$
\left|\operatorname{Dyck}_{n}(k, d)\right|=\frac{1}{n+1}\left(\left(\begin{array}{c}
n+1 \\
d
\end{array}\right)\right)\left(\left(\begin{array}{c}
k(n+1) \\
n-d
\end{array}\right)\right) .
$$

Proof. By Theorem 11,

$$
\left|\operatorname{Dyck}_{n}(k, d)\right|=\frac{1}{n+1}\left(\left(\begin{array}{c}
n+1 \\
d
\end{array}\right)\right) \sum_{a_{1}+\cdots+a_{k}=n-d} \prod_{i=1}^{k}\left(\left(\begin{array}{c}
n+1 \\
a_{i}
\end{array}\right)\right) .
$$

The above sum is equal to the number of $k$-tuples $\left(A_{1}, \ldots, A_{k}\right)$ of multisets such that each element $x \in A_{i}$ satisfies $(n+1)(i-1)+1 \leqslant x \leqslant(n+1) i$ and $\sum_{i=1}^{k}\left|A_{i}\right|=n-d$. Since such a $k$-tuple is completely determined by $A:=A_{1} \cup \cdots \cup A_{k}$, the sum is equal to $\left(\left(\begin{array}{c}k(n+1) \\ n-d\end{array}\right)\right)$, the number of multisets of size $n-d$ whose elements are in $[k(n+1)]$. Thus we obtain the formula. 
Corollary 18. We have

$$
\left|\operatorname{Dyck}_{n-1}^{(2)}(k)\right|=\frac{1}{k(n+1)+n-2}\left(\begin{array}{c}
k n+k+2 n-3 \\
n
\end{array}\right)\left(\begin{array}{c}
n+k-2 \\
k-1
\end{array}\right) .
$$

Proof. We will use the following standard notation in hypergeometric series:

$$
{ }_{2} F_{1}\left(\begin{array}{c}
a, b \\
c
\end{array} ; z\right)=\sum_{i \geqslant 0} \frac{(a)_{i}(b)_{i}}{(c)_{i}} \frac{z^{i}}{i !}
$$

where $(a)_{i}=a(a+1) \ldots(a+i-1)$. By Proposition 8 and Corollary 17,

$$
\begin{aligned}
\left|\operatorname{Dyck}_{n-1}^{(2)}(k)\right| & =\sum_{d=0}^{n-1}\left|\operatorname{Dyck}_{n-1}(k, d)\right|\left(\left(\begin{array}{c}
k \\
d+n-1
\end{array}\right)\right) \\
& =\sum_{d=0}^{n-1} \frac{1}{n}\left(\left(\begin{array}{c}
k n \\
n-d-1
\end{array}\right)\right)\left(\left(\begin{array}{l}
n \\
d
\end{array}\right)\right)\left(\left(\begin{array}{c}
k \\
d+n-1
\end{array}\right)\right) \\
& =\frac{(k n+n-2) !(k+n-2) !}{n !(k n-1) !(n-1) !(k-1) !}{ }_{2} F_{1}\left(\begin{array}{c}
-n+1, k+n-1 \\
-k n-n+2
\end{array} ; 1\right) .
\end{aligned}
$$

By the Vandermonde summation formula [1, Corollary 2.2.3]

$$
{ }_{2} F_{1}\left(\begin{array}{c}
-n, b \\
c
\end{array} ; 1\right)=\frac{(c-b)_{n}}{(c)_{n}},
$$

we have

$$
\begin{aligned}
{ }_{2} F_{1}\left(\begin{array}{c}
-n+1, k+n-1 \\
-k n-n+2
\end{array} 1\right) & =\frac{(-k n-2 n-k+3)_{n-1}}{(-k n-n+2)_{n-1}} \\
& =\frac{(k n+2 n+k-3) !}{(k n+n+k-2) !} \frac{(k n-1) !}{(k n+n-2) !} .
\end{aligned}
$$

By (17) and the above equation, we obtain the result.

The constant term identities in Theorems 6 and 7 follow immediately from Theorems 9 , 10 and Corollary 18. This completes the proof of Theorems 1 and 2 in the introduction.

\section{More Properties of Labeled Dyck Paths}

In this section we find volumes of flow polytopes of Pitman-Stanley graph $\mathrm{PS}_{n+1}$ and caracol graph $\mathrm{Car}_{n+1}$ for certain flow vectors using Lidskii's formula and $k$-labeled Dyck prefixes.

A $k$-labeled Dyck prefix is the part of a $k$-labeled Dyck path from $(0,0)$ to $(a, b)$ for some point $(a, b)$ in the path. The set of $k$-labeled Dyck prefixes from $(0,0)$ to $(2 n-i, i)$ is 


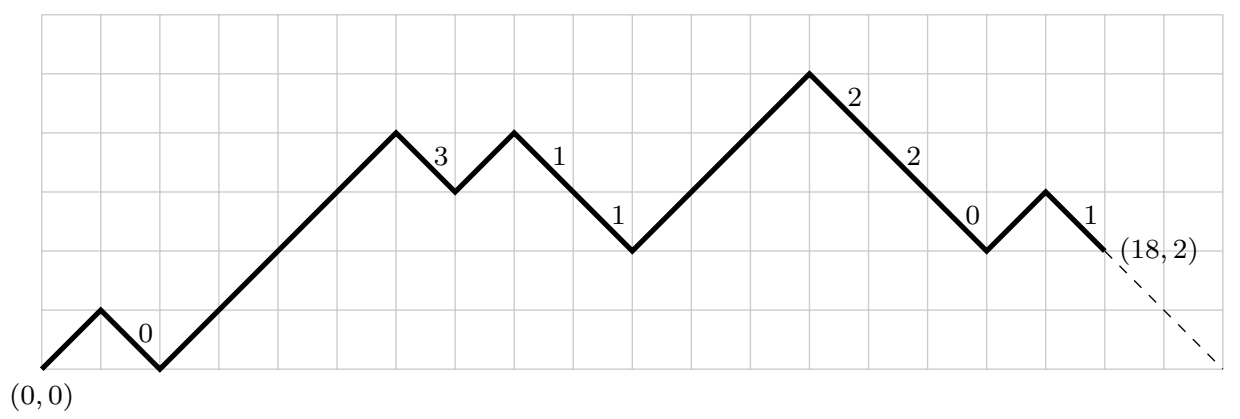

Figure 5: An element of $\operatorname{Dyck}_{20,2}(3 ; 2,3,2,1)$ whose steps are drawn in solid segments. By appending it with the two dashed down-steps, this element can be considered as an element in $\operatorname{Dyck}_{20}(3)$. This labeled Dyck path can be expressed as $U D_{0} U U U U D_{3} U D_{1} D_{1} U U U D_{2} D_{2} D_{0} U D_{1} D_{0} D_{0}$, where the last two $D_{0}$ steps correspond to the dashed down-steps.

denoted by Dyck ${ }_{n, i}$. We also denote by $\operatorname{Dyck}_{n, i}\left(k ; a_{0}, a_{1}, \ldots, a_{k}\right)$ the set of $k$-labeled Dyck prefixes in Dyck ${ }_{n, i}$ such that the number of down-steps labeled $j$ is $a_{j}$ for $0 \leqslant j \leqslant k$.

Recall that $\operatorname{Dyck}_{n}(k)$ is in bijection with the set of $k$-Dyck words of length $2 n$. Therefore one can consider an element in Dyck $_{n, i}$ as a $k$-Dyck word of length $2 n$ whose last $i$ letters are $D_{0}$ 's. See Figure 5 .

Now we find the cardinality of $\operatorname{Dyck}_{n, i}\left(k ; a_{0}, a_{1}, \ldots, a_{k}\right)$.

Lemma 19. We have

$$
\left|\operatorname{Dyck}_{n, i}\left(k ; a_{0}, a_{1}, \ldots, a_{k}\right)\right|=\frac{i+1}{n+1} \prod_{j=0}^{k}\left(\left(\begin{array}{c}
n+1 \\
a_{j}
\end{array}\right)\right) .
$$

Proof. Let $\mathrm{EW}_{n, i}(k)$ be the set of words $w=w_{1} \ldots w_{2 n-i+1}$ of letters in $\left\{U, D_{0}, \ldots, D_{k}\right\}$ with $w_{1}=U$ and exactly $n+1 U$ 's that satisfies the third condition of a $k$-labeled Dyck word: the labels of any consecutive $D_{i}$ 's are in weakly decreasing order, i.e., if $w_{i}=D_{a}$ and $w_{i+1}=D_{b}$, then $a \geqslant b$. For $w \in \mathrm{EW}_{n, i}(k)$, an index candidate of $w$ is an integer $j$ satisfying the following condition:

- Find a letter $U$ followed by a $D_{i}$ for some $0 \leqslant i \leqslant k$ in cyclic order and delete this pair $U$ and $D_{i}$ from $w$. Repeat this until there are $i+1$ letters left, which must be all $U$ 's. Then the $j$ th $U$ in the original word $w$ is one of the remaining $U$ 's.

Note that there are $i+1$ index candidates for any $w \in \mathrm{EW}_{n, i}(k)$.

Let $\mathrm{EW}_{n, i}^{\prime}(k)$ be the set of words obtained from a word $w \in \mathrm{EW}_{n, i}(k)$ by adding $i D_{0}$ 's to the left of the $j$ th $U$ in $w$ for an index candidate $j$ of $w$. Note that $\mathrm{EW}_{n, i}^{\prime}(k)$ is a subset of $\operatorname{EW}_{n}(k)$ which is defined in Section 4. Thus every $w^{\prime} \in \mathrm{EW}_{n, i}^{\prime}(k)$ has length $2 n+1$ and the unique index index $\left(w^{\prime}\right)$ exists. Then by the map $p$ defined in Proposition 16, there is an $(n+1)$-to-1 map from $\mathrm{EW}_{n, i}^{\prime}(k)$ to $\operatorname{Dyck}_{n, i}(k)$. Since there are $(i+1)$ ways to choose 
an index candidate for $w \in \mathrm{EW}_{n, i}(k)$, we have

$$
(i+1) \prod_{j=0}^{k}\left(\left(\begin{array}{c}
n+1 \\
a_{j}
\end{array}\right)\right)=(n+1)\left|\operatorname{Dyck}_{n, i}\left(k ; a_{0}, a_{1}, \ldots, a_{k}\right)\right|,
$$

which completes the proof.

\subsection{Volumes of flow polytopes for the Pitman-Stanley graph.}

Recall that vol $\mathcal{F}_{\mathrm{PS}_{n+1}}\left(a^{n}\right)$ and vol $\mathcal{F}_{\mathrm{PS}_{n+1}}\left(a, b^{n-1}\right)$ were computed in [3] and [13]. In this subsection using Lemma 19 we compute vol $\mathcal{F}_{\mathrm{PS}_{n+1}}\left(a_{1}, \ldots, a_{k}, b^{n-k}\right)$ for $0 \leqslant k \leqslant 3$. For simplicity, we will consider $\mathrm{PS}_{n+2}$ instead of $\mathrm{PS}_{n+1}$.

Note that $\mathrm{PS}_{n+2}$ has $2 n+1$ edges and $\mathbf{t}:=(\operatorname{outdeg}(1)-1, \ldots, \operatorname{outdeg}(n+1)-1)=$ $(1,1, \ldots, 1,0)$. Since $\left.\mathrm{PS}_{n+2}\right|_{n+1}$ is the path graph on $[n+1]$ with edges $(i, i+1)$ for $1 \leqslant i \leqslant n$, one can easily see that $K_{\left.\mathrm{PS}_{n+2}\right|_{n+1}}(\mathbf{s}-\mathbf{t})=1$ for any sequence $\mathbf{s} \geqslant \mathbf{t}$. Moreover, if $\mathbf{s}=\left(s_{1}, \ldots, s_{n+1}\right) \geqslant \mathbf{t}$, then $s_{n+1}=0$. Thus Lidskii's formula (Theorem 3) implies

$$
\operatorname{vol} \mathcal{F}_{\mathrm{PS}_{n+2}}\left(a_{1}, \ldots, a_{n+1}\right)=\sum_{\substack{s_{1}+\cdots+s_{n}=n \\
\left(s_{1}, \ldots, s_{n}\right) \geqslant\left(1^{n}\right)}}\left(\begin{array}{c}
n \\
s_{1}, s_{2}, \ldots, s_{n}
\end{array}\right) a_{1}^{s_{1}} \ldots a_{n}^{s_{n}} .
$$

Thus, we have

$$
\begin{aligned}
& \operatorname{vol} \mathcal{F}_{\mathrm{PS}_{n+2}}\left(a_{1}, \ldots, a_{k}, b^{n-k+1}\right) \\
& =\sum_{\substack{s_{1}+\cdots+s_{n}=n \\
\left(s_{1}, \ldots, s_{n}\right) \geqslant\left(1^{n}\right)}}\left(\begin{array}{c}
n \\
s_{1}, s_{2}, \ldots, s_{n}
\end{array}\right) a_{1}^{s_{1}} \ldots a_{k}^{s_{k}} b^{s_{k+1}+\cdots+s_{n}} \\
& =\sum_{m=0}^{n} \sum_{\substack{s_{1}+\cdots+s_{k}=m \\
\left(s_{1}, \ldots, s_{k}\right) \geqslant\left(1^{k}\right)}} \sum_{\substack{s_{k+1}+\cdots+s_{n}=n-m \\
\left(m, s_{k+1}, \ldots, s_{n}\right) \geqslant\left(k, 1^{n-k}\right)}}\left(\begin{array}{c}
n \\
s_{1}, s_{2}, \ldots, s_{n}
\end{array}\right) a_{1}^{s_{1}} \ldots a_{k}^{s_{k}} b^{n-m} \\
& =\sum_{m=0}^{n}\left(\begin{array}{c}
n \\
m
\end{array}\right) b^{n-m} A_{k, m}\left(a_{1}, \ldots, a_{k}\right) B_{n, k, m}
\end{aligned}
$$

where

$$
\begin{aligned}
A_{k, m}\left(a_{1}, \ldots, a_{k}\right)= & \sum_{\substack{s_{1}+\cdots+s_{k}=m \\
\left(s_{1}, \ldots, s_{k}\right) \geqslant\left(1^{k}\right)}}\left(\begin{array}{c}
m \\
s_{1}, s_{2}, \ldots, s_{k}
\end{array}\right) a_{1}^{s_{1}} \ldots a_{k}^{s_{k}} \\
B_{n, k, m}= & \sum_{\substack{s_{k+1}+\cdots+s_{n}=n-m \\
\left(m, s_{k+1}, \ldots, s_{n}\right) \geqslant\left(k, 1^{n-k}\right)}}\left(\begin{array}{c}
n-m \\
s_{k+1}, \ldots, s_{n}
\end{array}\right) .
\end{aligned}
$$

The following lemma shows that $B_{n, k, m}$ has a simple formula. 
Lemma 20. We have

$$
B_{n, k, m}=(m-k+1)(n-k+1)^{n-m-1} .
$$

Proof. For a sequence $\left(s_{k+1}, \ldots, s_{n}\right)$ of nonnegative integers, we have $s_{k+1}+\cdots+s_{n}=$ $n-m$ and $\left(m, s_{k+1}, \ldots, s_{n}\right) \geqslant\left(k, 1^{n-k}\right)$ if and only if $U D^{s_{n}} U D^{s_{n-1}} \ldots U D^{s_{k+1}} U^{k} D^{m}$ is a Dyck path from $(0,0)$ to $(2 n, 0)$, or equivalently, $U D^{s_{n}} U D^{s_{n-1}} \ldots U D^{s_{k+1}}$ is a Dyck prefix from $(0,0)$ to $(2 n-m-k, m-k)$. Moreover, if such a sequence $\left(s_{k+1}, \ldots, s_{n}\right)$ is given, $\left(\begin{array}{c}n-m \\ s_{k+1}, \ldots, s_{n}\end{array}\right)$ is the number of ways to label the down steps of this Dyck prefix with labels from $\{0,1, \ldots, n-m-1\}$ such that there is exactly one down step labeled $j$ for each $0 \leqslant j \leqslant n-m-1$ and the labels of consecutive down steps are in decreasing order. Thus

$$
B_{n, k, m}=\left|\operatorname{Dyck}_{n-k, m-k}\left(n-m-1 ; 1^{n-m}\right)\right| .
$$

By Lemma 19 we obtain the formula.

By (18) and Lemma 20, we obtain the following proposition.

Proposition 21. We have

$$
\begin{aligned}
& \operatorname{vol} \mathcal{F}_{\mathrm{PS}_{n+2}}\left(a_{1}, \ldots, a_{k}, b^{n-k+1}\right) \\
& =\sum_{m=0}^{n}\left(\begin{array}{c}
n \\
m
\end{array}\right) b^{n-m}(m-k+1)(n-k+1)^{n-m-1} A_{k, m}\left(a_{1}, \ldots, a_{k}\right),
\end{aligned}
$$

where

$$
A_{k, m}\left(a_{1}, \ldots, a_{k}\right)=\sum_{\substack{s_{1}+\cdots+s_{k}=m \\
\left(s_{1}, \ldots, s_{k}\right) \geqslant\left(1^{k}\right)}}\left(\begin{array}{c}
m \\
s_{1}, s_{2}, \ldots, s_{k}
\end{array}\right) a_{1}^{s_{1}} \ldots a_{k}^{s_{k}}
$$

By Proposition 21, in order to compute vol $\mathcal{F}_{\mathrm{PS}_{n+2}}\left(a_{1}, \ldots, a_{k}, b^{n-k+1}\right)$, it is enough to find $A_{k, m}\left(a_{1}, \ldots, a_{k}\right)$. For $k=0,1$, using this method we can easily recover the following formulas in $[3,13]$ :

$$
\begin{aligned}
& \operatorname{vol} \mathcal{F}_{\mathrm{PS}_{n+2}}\left(a^{n+1}\right)=a^{n}(n+1)^{n-1}, \\
& \operatorname{vol} \mathcal{F}_{\mathrm{PS}_{n+2}}\left(a, b^{n}\right)=a(a+n b)^{n-1} .
\end{aligned}
$$

We now find a formula for this volume for vol $\mathcal{F}_{\mathrm{PS}_{n+2}}\left(a_{1}, \ldots, a_{k}, b^{n-k+1}\right)$ for $k=2,3$.

Proposition 22. For positive integers $a, b$, and $c$, we have

$$
\operatorname{vol} \mathcal{F}_{\mathrm{PS}_{n+2}}\left(a, b, c^{n-1}\right)=(a+b-c)(a+b+(n-1) c)^{n-1}-(b-c)(b+(n-1) c)^{n-1} .
$$

Proof. By Proposition 21,

$$
\operatorname{vol} \mathcal{F}_{\mathrm{PS}_{n+2}}\left(a, b, c^{n-1}\right)=\sum_{m=0}^{n}\left(\begin{array}{c}
n \\
m
\end{array}\right) c^{n-m}(m-1)(n-1)^{n-m-1} A_{2, m}(a, b),
$$


where $A_{2,0}(a, b)=A_{2,1}(a, b)=0$ and for $m>2$,

$$
A_{2, m}(a, b)=\sum_{\substack{i+j=m \\
(i, j) \geqslant(1,1)}}\left(\begin{array}{c}
m \\
i, j
\end{array}\right) a^{i} b^{j}=(a+b)^{m}-b^{m} .
$$

Thus

$$
\begin{aligned}
& \operatorname{vol} \mathcal{F}_{\mathrm{PS}_{n+2}}\left(a, b, c^{n-1}\right)= \sum_{m=2}^{n}\left(\begin{array}{l}
n \\
m
\end{array}\right) c^{n-m}\left((a+b)^{m}-b^{m}\right)(m-1)(n-1)^{n-m-1} \\
&= \frac{1}{n-1}\left(g_{n}(a+b, c(n-1))-g_{n}(b, c(n-1))\right. \\
&\left.\quad-f_{n}(a+b, c(n-1))+f_{n}(b, c(n-1))\right),
\end{aligned}
$$

where

$$
\begin{aligned}
& f_{n}(x, y)=\sum_{m=0}^{n}\left(\begin{array}{l}
n \\
m
\end{array}\right) x^{m} y^{n-m}=(x+y)^{n} \\
& g_{n}(x, y)=\sum_{m=0}^{n} m\left(\begin{array}{c}
n \\
m
\end{array}\right) x^{m} y^{n-m}=n x(x+y)^{n-1} .
\end{aligned}
$$

Simplifying (19) we obtain the result.

In a similar way one can check $A_{3, m}(a, b, c)=(a+b+c)^{m}-(b+c)^{m}-a c^{m-1}$ and obtain the following proposition. We omit the details.

Proposition 23. For positive integers $a, b, c$, and $d$, we have

$$
\begin{aligned}
\operatorname{vol} \mathcal{F}_{\mathrm{PS}_{n+2}}\left(a, b, c, d^{n-2}\right) & =(a+b+c-2 d)(a+b+c+(n-2) d)^{n-1} \\
& -(b+c-2 d)(b+c+(n-2) d)^{n-1}-n a(c-d)(c+(n-2) d)^{n-2} .
\end{aligned}
$$

\subsection{Volumes of flow polytopes for the caracol graph.}

In [3], Benedetti et al. computed vol $\mathcal{F}_{\mathrm{Car}_{n+1}}\left(a^{n}\right)$ and $\operatorname{vol} \mathcal{F}_{\mathrm{Car}_{n+1}}\left(a, b^{n-1}\right)$ using unified diagrams and conjectured a formula for vol $\mathcal{F}_{\mathrm{Car}_{n+1}}\left(a, b, c^{n-2}\right)$, see Proposition 26 below. In this subsection we prove their conjecture. As before, for simplicity, we consider $\mathrm{Car}_{n+2}$ instead of $\mathrm{Car}_{n+1}$.

The caracol graph $\operatorname{Car}_{n+2}$ has $3 n-1$ edges and $\mathbf{t}^{\prime}:=(\operatorname{outdeg}(1)-1, \ldots, \operatorname{outdeg}(n+$ $1)-1)=(n-1,1,1, \ldots, 1,0)$. Note that $\mathbf{s}=\left(s_{1}, \ldots, s_{n+1}\right) \geqslant \mathbf{t}^{\prime}$ implies $s_{n+1}=0$. Thus, by Lidskii's formula,

$$
\begin{aligned}
& \operatorname{vol} \mathcal{F}_{\operatorname{Car}_{n+2}}\left(a_{1}, \ldots, a_{n+1}\right) \\
& =\sum_{\substack{s_{1}+\cdots+s_{n}=2 n-2 \\
\left(s_{1}, \ldots, s_{n}\right) \geqslant\left(n-1,1^{n-1}\right)}}\left(\begin{array}{c}
2 n-2 \\
s_{1}, \ldots, s_{n}
\end{array}\right) a_{1}^{s_{1}} \ldots a_{n}^{s_{n}} K_{\left.\operatorname{Car}_{n+2}\right|_{n}}\left(\left(s_{1}, \ldots, s_{n}\right)-\left(n-1,1^{n-1}\right)\right) .
\end{aligned}
$$


Our goal is to find a formula for $X:=\operatorname{vol} \mathcal{F}_{\mathrm{Car}_{n+2}}\left(a, b, c^{n-1}\right)$. By the above equation,

$$
\begin{aligned}
X= & \sum_{\substack{s_{1}+\cdots+s_{n}=2 n-2 \\
\left(s_{1}, \ldots, s_{n}\right) \geqslant\left(n-1,1^{n-1}\right)}}\left(\begin{array}{c}
2 n-2 \\
s_{1}, s_{2}, \ldots, s_{n}
\end{array}\right) a^{s_{1}} b^{s_{2}} c^{s_{3}+\cdots+s_{n}} \\
& \times K_{\left.\operatorname{Car}_{n+2}\right|_{n}}\left(s_{1}-n+1, s_{2}-1, \ldots, s_{n}-1\right) .
\end{aligned}
$$

By replacing $s_{1}$ by $s_{1}+n-2$, we obtain

$$
\begin{aligned}
X=\sum_{\substack{s_{1}+\cdots+s_{n}=n \\
\left(s_{1}, \ldots, s_{n}\right) \geqslant\left(1^{n}\right)}}\left(\begin{array}{c}
2 n-2 \\
s_{1}+n-2, s_{2}, \ldots, s_{n}
\end{array}\right) a^{s_{1}+n-2} b^{s_{2}} c^{s_{3}+\cdots+s_{n}} & \\
& \times K_{\left.\mathrm{Car}_{n+2}\right|_{n}}\left(s_{1}-1, \ldots, s_{n}-1\right) .
\end{aligned}
$$

Considering $p=s_{1}, q=s_{2}$, and $r=s_{3}+\cdots+s_{n}$ separately, we can rewrite the above equation as

$$
X=\sum_{\substack{p+q+r=n \\
(p, q) \geqslant(1,1)}}\left(\begin{array}{c}
2 n-2 \\
p+n-2, q, r
\end{array}\right) a^{p+n-2} b^{q} c^{r} A(p, q, r)
$$

where

$$
A(p, q, r)=\sum_{\substack{s_{3}+\cdots+s_{n}=r \\
\left(p, q, s_{3}, \ldots, s_{n}\right) \geqslant\left(1^{n}\right)}}\left(\begin{array}{c}
r \\
s_{3}, \ldots, s_{n}
\end{array}\right) K_{\left.\mathrm{Car}_{n+2}\right|_{n}}\left(p-1, q-1, s_{3}-1, \ldots, s_{n}-1\right) .
$$

In the next two lemmas we find a formula for $A(p, q, r)$ using labeled Dyck paths.

Note that every Dyck path of length $2 n$ can be expressed uniquely as a sequence $U D^{d_{n}} U D^{d_{n-1}} \ldots U D^{d_{1}}$ of up steps $U$ and down steps $D$ for some $n$-tuple $\left(d_{1}, \ldots, d_{n}\right) \in \mathbb{Z}_{\geqslant 0}^{n}$ such that $d_{1}+\cdots+d_{n}=n$ and $\left(d_{1}, \ldots, d_{n}\right) \geqslant\left(1^{n}\right)$. For nonnegative integers $a_{1}, \ldots, a_{n}$ whose sum is at most $n$, let

$$
D_{n}\left(a_{1}, \ldots, a_{n}\right):=\left\{U D^{d_{n}} U D^{d_{n-1}} \ldots U D^{d_{1}} \in \operatorname{Dyck}_{n}: d_{i} \geqslant a_{i}\right\} .
$$

Lemma 24. Let $\left(s_{1}, \ldots, s_{n}\right) \in \mathbb{Z}_{\geqslant 0}^{n}$ with $\sum_{i=1}^{n} s_{i}=n$ and $\left(s_{1}, \ldots, s_{n}\right) \geqslant\left(1^{n}\right)$. Then

$$
K_{\left.\operatorname{Car}_{n+2}\right|_{n}}\left(s_{1}-1, \ldots, s_{n}-1\right)=\left|D_{n-1}\left(s_{2}, \ldots, s_{n}\right)\right| .
$$

Proof. Note that $\left.\mathrm{Car}_{n+2}\right|_{n}$ is a directed graph on $[n]$ with edges $(1, i)$ for $2 \leqslant i \leqslant$ $n$ and $(j, j+1)$ for $2 \leqslant j \leqslant n-1$. By definition of Kostant partition function, $K_{\left.\mathrm{Car}_{n+2}\right|_{n}}\left(\left(s_{1}, \ldots, s_{n}\right)-\left(1^{n}\right)\right)$ is the number of nonnegative integer solutions $\left\{b_{1, i}, b_{j, j+1}\right.$ : $2 \leqslant i \leqslant n, 2 \leqslant j \leqslant n-1\}$ satisfying

$$
\begin{aligned}
b_{1,2}+b_{1,3}+\cdots+b_{1, n} & =s_{1}-1, \\
b_{2,3}-b_{1,2} & =s_{2}-1, \\
b_{j, j+1}-b_{j-1, j}-b_{1, j} & =s_{j}-1, \quad(3 \leqslant j \leqslant n),
\end{aligned}
$$


where $b_{n, n+1}=0$. The above equations are equivalent to

$$
\begin{aligned}
b_{1,2}+b_{1,3}+\cdots+b_{1, n} & =s_{1}-1, \\
b_{j, j+1} & =\left(s_{2}+\cdots+s_{j}\right)+\left(b_{1,2}+\cdots+b_{1, j}\right)-(j-1), \quad(2 \leqslant j \leqslant n) .
\end{aligned}
$$

Thus the integers $b_{j, j+1}$ for $2 \leqslant j \leqslant n-1$ are completely determined by the integers $b_{1, i}$ for $2 \leqslant i \leqslant n$. Moreover, the condition $b_{j, j+1} \geqslant 0$ for $2 \leqslant j \leqslant n-1$ is equivalent to $\left(s_{2}, \ldots, s_{n}\right)+\left(b_{12}, \ldots, b_{1 n}\right) \geqslant\left(1^{n-1}\right)$ in dominance order. Hence $K_{\left.\operatorname{Car}_{n+2}\right|_{n}}\left(\left(s_{1}, \ldots, s_{n}\right)-\right.$ $\left.\left(1^{n}\right)\right)$ is the number of $(n-1)$-tuples $\left(b_{12}, b_{13}, \ldots, b_{1 n}\right) \in \mathbb{Z}_{\geqslant 0}^{n-1}$ such that $b_{12}+b_{13}+\cdots+b_{1 n}=$ $s_{1}-1$ and $\left(s_{2}, \ldots, s_{n}\right)+\left(b_{12}, \ldots, b_{1 n}\right) \geqslant\left(1^{n-1}\right)$.

Now let $d_{i}=s_{i+1}+b_{1, i+1}$ for $1 \leqslant i \leqslant n-1$. Then we can express $K_{\left.\mathrm{Car}_{n+2}\right|_{n}}\left(\left(s_{1}, \ldots, s_{n}\right)-\right.$ $\left.\left(1^{n}\right)\right)$ as the number of $(n-1)$-tuples $\left(d_{1}, \ldots, d_{n-1}\right) \in \mathbb{Z}_{\geqslant 0}^{n-1}$ such that $d_{1}+\cdots+d_{n-1}=$ $n-1,\left(d_{1}, \ldots, d_{n-1}\right) \geqslant\left(1^{n-1}\right)$ and $d_{i} \geqslant s_{i+1}$ for $1 \leqslant i \leqslant n-1$. Since the condition $\left(d_{1}, \ldots, d_{n-1}\right) \geqslant\left(1^{n-1}\right)$ is equivalent to the condition $U D^{d_{n-1}} U D^{d_{n-2}} \cdots U D^{d_{1}} \in$ Dyck $_{n-1}$, we obtain the desired result.

Lemma 25. Let $p, q$ and $r$ be fixed nonnegative integers with $p+q+r=n$ and $(p, q) \geqslant$ $(1,1)$. Then

$$
A(p, q, r)=(p+q-1)\left(\begin{array}{c}
n+p-2 \\
n-1
\end{array}\right)(n-1)^{r-1}-\left(\begin{array}{c}
n+p-2 \\
n
\end{array}\right)(n-1)^{r} .
$$

Proof. By Lemma 24,

$$
A(p, q, r)=\sum_{\substack{s_{3}+\cdots+s_{n}=r \\
\left(p, q, s_{3}, \ldots, s_{n}\right) \geqslant\left(1^{n}\right)}}\left(\begin{array}{c}
r \\
s_{3}, \ldots, s_{n}
\end{array}\right)\left|D_{n-1}\left(q, s_{3}, \ldots, s_{n}\right)\right| .
$$

We will give a combinatorial interpretation of each summand in the above formula using labeled Dyck paths. Let $s_{3}, \ldots, s_{n}$ be nonnegative integers satisfying $s_{3}+\cdots+s_{n}=$ $r$ and $\left(p, q, s_{3}, \ldots, s_{n}\right) \geqslant\left(1^{n}\right)$. Consider a Dyck path $\pi=U D^{d_{n-1}} U D^{d_{n-2}} \ldots U D^{d_{1}} \in$ $D_{n-1}\left(q, s_{3}, \ldots, s_{n}\right)$. Then $d_{1} \geqslant q$ and $d_{i} \geqslant s_{i+1}$ for $2 \leqslant i \leqslant n-1$. Now we label the down steps of $\pi$ except the last consecutive down steps $D^{d_{1}}$ as follows:

- Distribute the $r$ labels $1,2, \ldots, r$, each label occurring exactly once, to the sequences $D^{d_{n-1}}, D^{d_{n-2}}, \ldots, D^{d_{2}}$ consecutive down steps of $\pi$ so that the sequence $D^{d_{i}}$ gets $s_{i+1}$ labels. There are $\left(\begin{array}{c}r \\ s_{3}, \ldots, s_{n}\end{array}\right)$ ways to do this.

- Add $d_{i}-s_{i+1}$ zero labels to the sequence $D^{d_{i}}$ and arrange the labels in weakly decreasing order.

By this process, we obtain that $\left(\begin{array}{c}r \\ s_{3}, \ldots, s_{n}\end{array}\right)\left|D_{n-1}\left(q, s_{3}, \ldots, s_{n}\right)\right|$ is the number of Dyck paths $\pi=U D^{d_{n-1}} U D^{d_{n-2}} \ldots U D^{d_{1}}$ together with a labeling on the down steps except the last consecutive down steps $D^{d_{1}}$ satisfying the following conditions:

1. $d_{i} \geqslant s_{i+1}$ for $2 \leqslant i \leqslant n-1$. 
2. $q \leqslant d_{1} \leqslant n-1-r$.

3. The number of down steps labeled $i$ is 1 for $1 \leqslant i \leqslant r$.

4. The number of down steps labeled 0 is $n-1-r-d_{1}$.

5. The labels of any consecutive down steps are weakly decreasing.

Summing over all possible $s_{3}, \ldots, s_{n}$ we obtain that $A(p, q, r)$ is the number of Dyck paths $\pi=U D^{d_{n-1}} U D^{d_{n-2}} \ldots U D^{d_{1}}$ together with a labeling on the down steps of its prefix $U D^{d_{n-1}} U D^{d_{n-2}} \ldots U D^{d_{2}}$ from $(0,0)$ to $\left(2 n-3-d_{1}, d_{1}-1\right)$ satisfying the above conditions except (1). This implies

$$
A(p, q, r)=\sum_{d_{1}=q}^{n-1-r}\left|\operatorname{Dyck}_{n-2, d_{1}-1}\left(r ; n-1-r-d_{1}, 1^{r}\right)\right| .
$$

By Lemma 19,

$$
\begin{aligned}
A(p, q, r) & =\sum_{d_{1}=q}^{n-1-r} \frac{d_{1}}{n-1}\left(\left(\begin{array}{c}
n-1 \\
n-1-r-d_{1}
\end{array}\right)\right)(n-1)^{r} \\
& =(n-1)^{r-1} \sum_{d_{1}=q}^{n-1-r} d_{1}\left(\begin{array}{c}
2 n-3-r-d_{1} \\
n-2
\end{array}\right) .
\end{aligned}
$$

Replacing $d_{1}$ by $n-1-r-i$, we have

$$
A(p, q, r)=(n-1)^{r-1} \sum_{i=0}^{p-1}(n-1-r-i)\left(\begin{array}{c}
n-2+i \\
n-2
\end{array}\right) .
$$

Since

$$
\begin{aligned}
(n-1-r-i)\left(\begin{array}{c}
n-2+i \\
n-2
\end{array}\right) & =((2 n-2-r)-(n-1+i))\left(\begin{array}{c}
n-2+i \\
n-2
\end{array}\right) \\
& =(2 n-2-r)\left(\begin{array}{c}
n-2+i \\
n-2
\end{array}\right)-(n-1)\left(\begin{array}{c}
n-1+i \\
n-1
\end{array}\right) \\
& =(n-1-r)\left(\begin{array}{c}
n-2+i \\
n-2
\end{array}\right)-(n-1)\left(\begin{array}{c}
n-2+i \\
n-1
\end{array}\right),
\end{aligned}
$$

we have

$$
A(p, q, r)=(n-1)^{r-1}\left((p+q-1) \sum_{i=0}^{p-1}\left(\begin{array}{c}
n-2+i \\
n-2
\end{array}\right)-(n-1) \sum_{i=0}^{p-2}\left(\begin{array}{c}
n-1+i \\
n-1
\end{array}\right)\right) .
$$

Finally the identity $\sum_{i=0}^{k}\left(\begin{array}{c}m+i \\ m\end{array}\right)=\left(\begin{array}{c}m+k+1 \\ m+1\end{array}\right)$ finishes the proof. 
Now we are ready to compute $X=\operatorname{vol} \mathcal{F}_{\operatorname{Car}_{n+2}}\left(a, b, c^{n-1}\right)$.

Proposition 26. [3, Conjecture 6.16] For positive integers $a, b$, and $c$, we have

$$
\operatorname{vol} \mathcal{F}_{\mathrm{Car}_{n+2}}\left(a, b, c^{n-1}\right)=C_{n-1} a^{n-1}(a+n b)(a+b+(n-1) c)^{n-2} .
$$

Proof. By (20) and Lemma 25, we have

$$
\operatorname{vol} \mathcal{F}_{\operatorname{Car}_{n+2}}\left(a, b, c^{n-1}\right)=X=Y-Z
$$

where

$$
\begin{gathered}
Y=\sum_{\substack{p+q+r=n \\
(p, q) \geqslant(1,1)}}\left(\begin{array}{c}
2 n-2 \\
p+n-2, q, r
\end{array}\right) a^{p+n-2} b^{q} c^{r}(p+q-1)\left(\begin{array}{c}
n+p-2 \\
n-1
\end{array}\right)(n-1)^{r-1}, \\
Z=\sum_{\substack{p+q+r=n \\
(p, q) \geqslant(1,1)}}\left(\begin{array}{c}
2 n-2 \\
p+n-2, q, r
\end{array}\right) a^{p+n-2} b^{q} c^{r}\left(\begin{array}{c}
n+p-2 \\
n
\end{array}\right)(n-1)^{r} .
\end{gathered}
$$

Note that in the above two sums, the condition $(p, q) \geqslant(1,1)$ can be omitted since the summand is zero if $p=0$ or $(p, q)=(1,0)$. Thus

$$
\begin{aligned}
Y & =\frac{a^{n-1}}{n-1}\left(\begin{array}{c}
2 n-2 \\
n-1
\end{array}\right) \sum_{p+q+r=n}(p+q-1)\left(\begin{array}{c}
n-1 \\
p-1, q, r
\end{array}\right) a^{p-1} b^{q}(c(n-1))^{r}, \\
Z & =\sum_{p+q+r=n}\left(\begin{array}{c}
2 n-2 \\
p+n-2, q, r
\end{array}\right) a^{p+n-2} b^{q} c^{r}\left(\begin{array}{c}
n+p-2 \\
n
\end{array}\right)(n-1)^{r} \\
& =a^{n}\left(\begin{array}{c}
2 n-2 \\
n
\end{array}\right) \sum_{p+q+r=n}\left(\begin{array}{c}
n-2 \\
p-2, q, r
\end{array}\right) a^{p-2} b^{q}(c(n-1))^{r} .
\end{aligned}
$$

Using the multinomial theorem

$$
\sum_{i+j+k=m}\left(\begin{array}{c}
m \\
i, j, k
\end{array}\right) x^{i} y^{j} z^{k} t^{i+j}=(x t+y t+z)^{m}
$$

and its derivative with respect to $t$, i.e,

$$
\sum_{i+j+k=m}(i+j)\left(\begin{array}{c}
m \\
i, j, k
\end{array}\right) x^{i} y^{j} z^{k} t^{i+j-1}=m(x+y)(x t+y t+z)^{m-1}
$$

we obtain

$$
\begin{aligned}
& Y=C_{n-1} a^{n-1} n(a+b)(a+b+(n-1) c)^{n-2}, \\
& Z=C_{n-1} a^{n}(n-1)(a+b+(n-1) c)^{n-2},
\end{aligned}
$$

and the proof follows. 


\section{Acknowledgments}

The authors would like to thank Alejandro Morales for informing them that Theorems 1 and 2 are equivalent to Theorems 6 and 7. They also thank Nathan Williams for helpful discussions. They are grateful to the anonymous referees for helpful comments.

\section{References}

[1] G. E. Andrews, R. Askey, and R. Roy. Special functions, volume 71 of Encyclopedia of Mathematics and its Applications. Cambridge University Press, Cambridge, 1999.

[2] W. Baldoni and M. Vergne. Kostant partitions functions and flow polytopes. Transform. Groups, 13(3-4):447-469, 2008.

[3] C. Benedetti, R. S. González D’León, C. R. H. Hanusa, P. E. Harris, A. Khare, A. H. Morales, and M. Yip. A combinatorial model for computing volumes of flow polytopes. Trans. Amer. Math. Soc., 372(5):3369-3404, 2019.

[4] C. S. Chan, D. P. Robbins, and D. S. Yuen. On the volume of a certain polytope. Experiment. Math., 9(1):91-99, 2000.

[5] S. Corteel, J. S. Kim, and K. Mészáros. Flow polytopes with Catalan volumes. C. R. Math. Acad. Sci. Paris, 355(3):248-259, 2017.

[6] K. Mészáros. Product formulas for volumes of flow polytopes. Proc. Amer. Math. Soc., 143(3):937-954, 2015.

[7] K. Mészáros and A. H. Morales. Volumes and Ehrhart polynomials of flow polytopes. arXiv: 1710.00701.

[8] K. Mészáros, A. H. Morales, and B. Rhoades. The polytope of Tesler matrices. Selecta Math. (N.S.), 23(1):425-454, 2017.

[9] K. Mészáros, A. H. Morales, and J. Striker. On flow polytopes, order polytopes, and certain faces of the alternating sign matrix polytope. Discrete Comput. Geom., 62(1):128-163, 2019.

[10] K. Mészáros, C. Simpson, and Z. Wellner. Flow polytopes of partitions. Electron. J. Combin., 26(1):\#P1.47, 2019.

[11] W. G. Morris. Constant term identities for finite and affine root systems: conjectures and theorems. PhD thesis, The University of Wisconsin - Madison, 1982.

[12] A. Selberg. Remarks on a multiple integral. Norsk Mat. Tidsskr., 26:71-78, 1944.

[13] R. P. Stanley and J. Pitman. A polytope related to empirical distributions, plane trees, parking functions, and the associahedron. Discrete Comput. Geom., 27(4):603$634,2002$.

[14] M. Yip. A Fuss-Catalan variation of the caracol flow polytope. arXiv:1910.10060.

[15] D. Zeilberger. Proof of a conjecture of Chan, Robbins, and Yuen. Electron. Trans. Numer. Anal., 9:147-148 (electronic), 1999. Orthogonal polynomials: numerical and symbolic algorithms (Leganés, 1998). 